\title{
ARTICLE OPEN \\ Cognitive subtypes in recent onset psychosis: distinct neurobiological fingerprints?
}

Julian Wenzel' ${ }^{1}$, Shalaila S. Haas ${ }^{2}$, Dominic B. Dwyer ${ }^{3}$, Anne Ruef ${ }^{3}$, Oemer Faruk Oeztuerk ${ }^{3,4}$, Linda A. Antonucci ${ }^{3,5}$, Sebastian von Saldern ${ }^{3}$, Carolina Bonivento ${ }^{6}$, Marco Garzitto ${ }^{6}$, Adele Ferro ${ }^{7,8}$, Marco Paolini ${ }^{9}$, Janusch Blautzik ${ }^{10}$, Stefan Borgwardt iD $^{11}$, Paolo Brambilla (iD ${ }^{7,8}$, Eva Meisenzahl ${ }^{12}$, Raimo K. R. Salokangas (iD ${ }^{13}$, Rachel Upthegrove ${ }^{14,15}$, Stephen J. Wood ${ }^{14,16,17}$, Joseph Kambeitz (D), Nikolaos Koutsouleris ${ }^{3,18,19}$, Lana Kambeitz-llankovic ${ }^{1,3}$ and the PRONIA consortium

In schizophrenia, neurocognitive subtypes can be distinguished based on cognitive performance and they are associated with neuroanatomical alterations. We investigated the existence of cognitive subtypes in shortly medicated recent onset psychosis patients, their underlying gray matter volume patterns and clinical characteristics. We used a K-means algorithm to cluster 108 psychosis patients from the multi-site EU PRONIA (Prognostic tools for early psychosis management) study based on cognitive performance and validated the solution independently $(N=53)$. Cognitive subgroups and healthy controls (HC; $n=195)$ were classified based on gray matter volume (GMV) using Support Vector Machine classification. A cognitively spared $(N=67)$ and impaired $(N=41)$ subgroup were revealed and partially independently validated $\left(N_{\text {spared }}=40, N_{\text {impaired }}=13\right)$. Impaired patients showed significantly increased negative symptomatology $\left(p_{\mathrm{fdr}}=0.003\right)$, reduced cognitive performance $\left(p_{\mathrm{fdr}}<0.001\right)$ and general functioning $\left(p_{\mathrm{fdr}}<0.035\right)$ in comparison to spared patients. Neurocognitive deficits of the impaired subgroup persist in both discovery and validation sample across several domains, including verbal memory and processing speed. A GMV pattern (balanced accuracy $=60.1 \%, p=0.01$ ) separating impaired patients from HC revealed increases and decreases across several fronto-temporalparietal brain areas, including basal ganglia and cerebellum. Cognitive and functional disturbances alongside brain morphological changes in the impaired subgroup are consistent with a neurodevelopmental origin of psychosis. Our findings emphasize the relevance of tailored intervention early in the course of psychosis for patients suffering from the likely stronger neurodevelopmental character of the disease.

Neuropsychopharmacology (2021) 46:1475-1483; https://doi.org/10.1038/s41386-021-00963-1

\section{INTRODUCTION}

In accordance with the neurodevelopmental hypothesis [1] the majority of patients suffering from psychosis show general and specific neurocognitive impairments $[2,3]$ as premorbid signs of early developmental insults and brain alterations [4]. However, studies report substantial heterogeneity regarding the severity of neurocognitive impairments [2] putatively representing different underlying disease trajectories marked by specific (neuro-) biological, clinical and functional characteristics [5].

Impaired cognitive and psychosocial functioning represent the top of the dysfunctional pyramid of schizophrenia (SZ) [6]. For a number of patients with psychosis, cognitive impairment persists beyond the presence of positive and negative symptoms and relates to reduced psychosocial outcome [6]. For this reason, identifying homogeneous subgroups of patients showing specific cognitive profiles may enhance the effects of promising novel treatments including neurocognitive interventions [7]. Previous studies using unsupervised machine learning (ML) found between two and four cognitive subgroups in SZ samples, ranging from unimpaired to severely deteriorated patient subgroups [8-11]. These subgroups differed not only with respect to their cognitive performance yet also in clinical symptomatology $[8,9,11]$, general $[8,10,11]$ and occupational functioning $[9,11]$. Furthermore, they were linked to different patterns of alterations in brain morphology [10, 12]. Complementary, studies using unsupervised ML identified neuroanatomical subgroups that were related to

\footnotetext{
${ }^{1}$ University of Cologne, Faculty of Medicine and University Hospital of Cologne, Cologne, Germany; ${ }^{2}$ Department of Psychiatry, Icahn School of Medicine at Mount Sinai, New York, NY, USA; ${ }^{3}$ Department of Psychiatry and Psychotherapy, Ludwig-Maximilian University, Munich, Germany; ${ }^{4}$ International Max Planck Research School for Translational Psychiatry, Max Planck Society, Munich, Germany; ${ }^{5}$ Department of Education, Psychology, Communication - University of Bari "Aldo Moro", Bari, Italy; ${ }^{6}$ Scientific Institute, IRCCS Eugenio

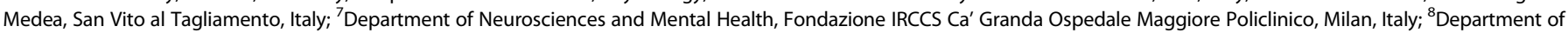
Pathophysiology and Transplantation, University of Milan, Milan, Italy; ${ }^{9}$ Department of Radiology, University Hospital, Ludwig-Maximilian University, Munich, Germany; ${ }^{10}$ Institute for Radiology and Nuclear Medicine St. Anna, Luzern, Switzerland; ${ }^{11}$ Translational Psychiatry Unit (TPU), Department of Psychiatry and Psychotherapy, University of Luebeck, Luebeck, Germany; ${ }^{12}$ Department of Psychiatry and Psychotherapy, Medical Faculty, Heinrich-Heine University, Düsseldorf, Germany; ${ }^{13}$ Department of Psychiatry, University of

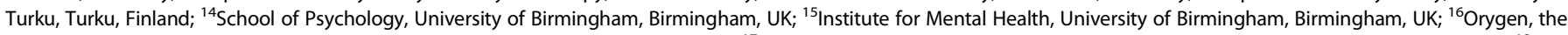
National Centre of Excellence for Youth Mental Health, Melbourne, VIC, Australia; ${ }^{17}$ Centre for Youth Mental Health, University of Melbourne, Melbourne, VIC, Australia; ${ }^{18}$ Max Planck Institute for Psychiatry, Munich, Germany and ${ }^{19}$ Institute of Psychiatry, Psychology and Neuroscience, King's College London, London, United Kingdom Correspondence: Lana Kambeitz-Ilankovic (lana.kambeitz-ilankovic@uk-koeln.de)
}

These authors contributed equally: Nikolaos Koutsouleris, Lana Kambeitz-Ilankovic.

A list of authors and their affiliations appears at the end of the paper.

Received: 25 September 2020 Revised: 16 December 2020 Accepted: 5 January 2021

Published online: 15 March 2021 
differences in premorbid functioning $[13,14]$ and neuropsychological performance [14].

Existing evidence on cognitive subgroups is mainly based on chronic SZ samples presenting with clinical symptoms for a prolonged period. These findings could be limited as patients may already be susceptible to change due to the effects of antipsychotic medication on cognitive performance [15] and brain structure [16].

The current study aims at disentangling variability in neurocognitive impairment. To achieve this, we (1) subgroup a recent onset psychosis (ROP) sample based on neurocognitive performance using cluster analysis and validate the cluster solution on neurocognitive data of an independent validation sample [17], (2) associate obtained ROP subgroups to symptom burden and functional disability and (3) investigate morphological brain differences between the cognitive subgroups and healthy controls $(\mathrm{HC})$ using gray matter volume (GMV) within a supervised $\mathrm{ML}$ framework.

\section{MATERIALS AND METHODS}

Sample

In the discovery sample 121 ROP patients and $201 \mathrm{HC}$, age between 15 and 40 years, were recruited within the PRONIA study (Personalized Prognostic tools for early psychosis management; www.pronia.eu; German Clinical Trials Register: DRKS00005042) at seven sites across Europe. Patients were included in the study if they fulfilled DSM-IV-TR criteria [18] for a psychotic episode present in the last 3 months, lasting longer than 1 week and with first onset in the last 24 months [19]. HC volunteers were required to not fulfill any current or past DSM-IV-TR axis I or II diagnosis, clinical high-risk (CHR) status for psychosis as defined by the Structured Interview for Prodromal Syndromes [20] and Schizophrenia Proneness Instrument [21] or positive familial history (1st degree relatives) for psychosis accompanied by a drop in functioning in the last year. HC participants with any intake of psychotropic medications more than five times/year or in the month before study entry were excluded. Written informed consent was obtained from the subjects. The study received ethical approval by each Local Research Ethics Committee at every study site separately (Supplementary Materials and Methods) [19].

The independent validation sample comprised baseline data of a monocentric, longitudinal cognitive intervention study called Personalized Neurocognitive Training (ClinicalTrials.gov Identifier: NCT03962426). Overall, 58 ROP patients were recruited at the Early Detection and Intervention Center at the Department of Psychiatry and Psychotherapy of the Ludwig-MaximiliansUniversity in Munich, Germany. Inclusion and exclusion criteria were identical to those required for the discovery sample of the PRONIA study.

The analysis data set consisted of 108 ROP patients and $195 \mathrm{HC}$ for the discovery sample and 53 ROP patients for the independent validation sample (Table 1, Fig. S8, Supplementary Materials and Methods).

\section{Clinical and neurocognitive assessment}

Participants were assessed using multiple clinical scales and neuropsychological tests focusing on the General Assessment of Functioning Scale (GAF) [22], split into two subscales (symptoms and disability), the Global Functioning Scale (GF social and occupational) [23] and the Positive and Negative Syndrome Scale (PANSS) [24]. The neuropsychological test battery comprised of ten tests that were assigned to cognitive domains comparable to the MATRICS Consensus Cognitive Battery (MCCB) domains [25] including visual memory (Rey-Osterrieth Complex Figure test [26]), social cognition (Diagnostic Analysis of Non-Verbal Accuracy [27]), working memory (Auditory Digit Span Task [28], Self-ordered Pointing Task [29]), processing speed (Verbal Fluency Test [30],
Trail Making Test A [31], Digit-Symbol-Substitution Test [28]), verbal learning and memory (Rey Auditory Verbal Learning Test [32]), executive functioning (Trail Making Test B [31]), attention and vigilance (Continuous Performance Test, Identical Pairs version [33]) and one psychosis-specific domain: aberrant salience [34] (Tables S1, S2 and Supplementary Materials and Methods).

Preprocessing and clustering of neurocognitive data

All selected neurocognitive variables were used. Preprocessing followed the steps of (1) imputing missing values by median and (2) linear regression of effects of age, sex, years of education and study site to account for site and demographic differences [35]. In addition, we used (3) principal component analyses (PCA) for dimensionality reduction on each group of neuropsychological variables associated with a certain cognitive domain (Table S1) and retained the first PCA component of each domain for cluster analysis (Fig. S1).

A K-means clustering algorithm [36] was applied to the neurocognitive domain values (PCA components) using Euclidean distance. Two independent resampling strategies were followed to assess cluster stability [37].

Preprocessing of the validation sample followed procedures identical to the discovery sample. To estimate the generalizability of the discovery clustering model to new observations, cluster assignment in the validation data set was based on the minimum Euclidean distance of a single observation to the centroids of the discovery sample cluster solution.

Demographic, clinical and neuropsychological characteristics of the obtained ROP subgroups and the $\mathrm{HC}$ sample were compared using one-way permutation and chi-squared tests. $P$ values were corrected using the Benjamini-Hochberg false discovery rate method [38] (Supplementary Materials and Methods).

Preprocessing, clustering and statistical analyses were conducted in R version 3.6.1 (https://cran.r-project.org/bin/windows/ base/). Cluster stability was assessed using the 'clusterboot'function [37] contained in the ' $\mathrm{fpc}^{\prime}$ package [39]. Cluster assignments of the validation observations were predicted using the 'flexclust' package [40]. Characteristics of subgroups were compared using non-parametric statistical tests from the 'coin'and the 'rcompanion'-package [41, 42].

Preprocessing of neuroimaging data

MRI data were inspected for scanner artefacts and anatomical abnormalities by a trained radiologist. Images were preprocessed using the open-source CAT12 toolbox (version > r1200; http:// dbm.neuro.uni-jena.de/cat12/), an extension of the SPM12 software (Wellcome Department of Cognitive Neurology, London, UK; http://www.fil.ion.ucl.ac.uk/spm/software/spm12/) following previously described steps [19] and the CAT12 manual (www.neuro.uni-jena.de/cat12/CAT12-Manual.pdf) (Supplementary Materials and Methods).

Neuroimaging classification analysis

A ML pipeline was employed to compare GMV between the obtained clusters and the $\mathrm{HC}$ population. Model generation and testing were embedded in a tenfold $\times$ tenfold nested crossvalidation pipeline with ten permutations on inner (CV1) and outer (CV2) loop using the in-house ML tool NeuroMiner (http:// www.pronia.eu/neurominer) running in MATLAB 2019a (MathWorks Inc.).

Within CV1 modulated, normalized GMV images were (1) smoothed with a Gaussian kernel (optimized for 4, 6 and $8 \mathrm{~mm}$ ), (2) corrected for total intracranial volume and (3) pruned by removing zero-variance voxels. Moreover, images were (4) pruned for voxels with low reliability across study sites using a $G$ coefficient map to account for scanner differences [19], (5) dimensionality was reduced by PCA (optimizing the retainment 
Table 1. Demographic and clinical characteristics of the discovery and validation sample used in the study.

\begin{tabular}{|c|c|c|c|c|c|c|c|c|c|}
\hline & \multicolumn{4}{|l|}{ Discovery } & \multicolumn{3}{|l|}{ Validation } & \multirow{2}{*}{\multicolumn{2}{|c|}{$\begin{array}{l}\text { Validation vs. } \\
\text { discovery } \\
\text { ROP (val) vs. } \\
\text { ROP (disc) }\end{array}$}} \\
\hline & \multirow[b]{2}{*}{$\operatorname{ROP}(N=108)$} & \multirow[b]{2}{*}{$\mathrm{HC}(N=195)$} & \multicolumn{2}{|c|}{ ROP vs. HC } & \multirow[b]{2}{*}{$\mathrm{ROP}(N=53)$} & \multicolumn{2}{|c|}{ ROP vs. HC } & & \\
\hline & & & $t / X^{2}$ & $p$ & & $t / X^{2}$ & $p$ & $t$ & $p$ \\
\hline \multicolumn{10}{|l|}{ Demographics } \\
\hline $\operatorname{Sex}^{\mathrm{a}}$ & Female $=35$ & Female $=121$ & 23.28 & $<0.001^{* * *}$ & Female $=21$ & 7.67 & $0.01 *$ & 0.53 & 0.47 \\
\hline Years of education & $14.08(3.3)$ & $16.02(3.43)$ & -4.83 & $<0.001^{* * *}$ & $14.05(3.54)$ & -3.62 & $<0.001^{* * *}$ & -0.06 & 0.96 \\
\hline Illness duration in days & $181.51(187.46)$ & - & - & - & $186.38(203.88)$ & - & - & -0.15 & 0.88 \\
\hline Chlorpromazine equivalent ${ }^{b}$ & $388.18(1020.61)$ & - & - & - & $1208.09(5205.17)$ & & & -1.06 & 0.29 \\
\hline \multicolumn{10}{|l|}{ Premorbid intelligence } \\
\hline Lifetime & 77.77 (10.09) & $88.48(5.63)$ & -10.15 & $<0.001^{* * *}$ & $77.22(8.79)$ & -8.61 & $<0.001^{* * *}$ & -0.35 & 0.73 \\
\hline Past year & $59.12(15.79)$ & $87.43(6.1)$ & -17.83 & $<0.001^{* * *}$ & $62.3(14.19)$ & -12.24 & $<0.001^{* * *}$ & 1.26 & 0.21 \\
\hline Past month & 41.85 (13.52) & $86.98(6.48)$ & -32.54 & $<0.001^{* * *}$ & $39.86(13.02)$ & -24.81 & $<0.001^{* * *}$ & -0.88 & 0.38 \\
\hline \multicolumn{10}{|l|}{ GAF (disability) } \\
\hline Lifetime & $77.11(8.99)$ & $86.84(5.21)$ & -10.29 & $<0.001^{* * *}$ & 75.78 (9.74) & -7.75 & $<0.001^{* * *}$ & -0.82 & 0.42 \\
\hline Past year & $61.36(13.66)$ & $85.95(5.82)$ & -17.76 & $<0.001^{* * *}$ & $61.82(14.21)$ & -11.76 & $<0.001^{* * *}$ & 0.19 & 0.85 \\
\hline Past month & $45.39(12.24)$ & $85.51(6.16)$ & -31.78 & $<0.001^{* * *}$ & $42.8(11.77)$ & -24.8 & $<0.001^{* * *}$ & -1.27 & 0.21 \\
\hline \multicolumn{10}{|l|}{ PANSS } \\
\hline Positive scale & $18.07(6.43)$ & - & - & - & $20.27(4.72)$ & - & - & -2.39 & $0.02^{*}$ \\
\hline Negative scale & $16.75(8.11)$ & - & - & - & $15.33(6.21)$ & - & - & 1.2 & 0.23 \\
\hline General scale & $36.05(10.6)$ & - & - & - & $34.02(10.02)$ & - & - & 1.15 & 0.25 \\
\hline
\end{tabular}

of the highest ranking components optimizing 40,60 and $80 \%)$ and (6) values were scaled between zero and one.

To find a discriminative pattern of GMV between groups, a linear support vector machine (SVM) algorithm (optimized cparameter range between 0.015625 and 16; 11 parameters) weighted by group sizes was applied on the GMV maps. Model performance was assessed by calculating the balanced accuracy (BAC). Statistical significance of the overall winning model was assessed using permutation tests $\left(N_{\text {perm }}=1000\right.$; alpha $\left.=0.05\right)$ [43]. Reliability of discriminative voxels contributing to the classification performance of the winning model was inspected by the cross-validation ratio (Supplementary Materials and Methods).

\section{RESULTS}

Discovery sample

A two-cluster solution indicated maxima on the Calinski-Harabasz index [44] and the average silhouette width score [45]. Stability assessment revealed clusterwise Jaccard similarity [46] indices of 0.84 and 0.90 for the 'subset' and 0.90 and 0.93 for the 'noise'-method, respectively, indicating highly stable clusters (Fig. S3) [37].
Neurocognitive characteristics

Patients in cluster $1(N=41)$ showed significantly lower performance in processing speed $\left(p_{\mathrm{fdr}}<0.001, d=1.89\right)$, executive functioning $\left(p_{\mathrm{fdr}}<0.001, d=-1.60\right)$, attention $\left(p_{\mathrm{fdr}}<0.001, d=\right.$ $1.01)$, working memory $\left(p_{\mathrm{fdr}}=0.004, d=0.67\right)$, verbal $\left(p_{\mathrm{fdr}}<0.001\right.$, $d=-1.37)$ and visual memory $\left(p_{\mathrm{fdr}}<0.001, d=1.44\right)$ as compared to patients belonging to cluster $2(N=67)$.

Cluster 1 patients showed significantly lower performance in processing speed $\left(p_{\mathrm{fdr}}<0.001, d=2.11\right)$, executive functioning $\left(p_{\mathrm{fdr}}<0.001, d=-0.77\right), \quad$ attention $\left(p_{\mathrm{fdr}}<0.001, \quad d=1.01\right)$, working memory $\left(p_{\mathrm{fdr}}<0.001, d=1.10\right)$ and verbal $\left(p_{\mathrm{fdr}}<0.001\right.$, $d=-2.43)$ and visual memory $\left(p_{\mathrm{fdr}}<0.001, d=1.66\right)$ as compared to HC group. We refer to cluster 1 as 'impaired' due to its largely inferior cognitive performance in comparison to cluster 2 and $\mathrm{HC}$.

Cluster 2 patients showed significantly decreased performance in attention $\left(p_{\mathrm{fdr}}<0.001, d=0.65\right)$ and verbal memory $\left(p_{\mathrm{fdr}}=\right.$ $0.001, d=-0.47$ ) as compared to HC. They showed improved performance in executive functioning $\left(p_{\mathrm{fdr}}<0.001, d=0.53\right)$, salience $\left(p_{\mathrm{fdr}}=0.003, d=0.44\right)$ and visual memory $\left(p_{\mathrm{fdr}}=0.003\right.$, $d=0.44$ ) compared to HC. We refer to this cluster as 'spared' as its performance was inferior to $\mathrm{HC}$ only in two cognitive domains (Table 2 and Fig. 1A). 
Table 2. Neuropsychological domain-specific effects between impaired and spared cluster and healthy controls in discovery and validation sample.

\begin{tabular}{|c|c|c|c|c|c|c|c|c|c|}
\hline & \multicolumn{3}{|l|}{ Overall } & \multicolumn{2}{|c|}{ Impaired vs. spared } & \multicolumn{2}{|c|}{ Impaired vs. HC } & \multicolumn{2}{|c|}{ Spared vs. HC } \\
\hline \multicolumn{10}{|l|}{ Discovery } \\
\hline Social cognition & 0.980 & 0.583 & 0.583 & - & - & - & - & - & - \\
\hline Working memory & 6.089 & $<0.001$ & $<0.001^{* * *}$ & $0.004^{* *}$ & 0.68 & $<0.001^{* * *}$ & 1.11 & 0.053 & 0.28 \\
\hline Processing speed & 10.070 & $<0.001$ & $<0.001^{* * *}$ & $<0.001^{* * *}$ & 1.90 & $<0.001^{* * *}$ & 2.12 & 0.223 & -0.17 \\
\hline Verbal memory & 10.385 & $<0.001$ & $<0.001^{* * *}$ & $<0.001^{* * *}$ & -1.39 & $<0.001^{* * *}$ & -2.44 & $0.001^{* *}$ & -0.48 \\
\hline Visual memory & 8.423 & $<0.001$ & $<0.001^{* * *}$ & $<0.001^{* * *}$ & 1.45 & $<0.001^{* * *}$ & 1.67 & $0.003^{* *}$ & -0.44 \\
\hline Salience & 2.646 & 0.022 & $0.023^{*}$ & 0.175 & -0.28 & 0.913 & -0.02 & $0.003^{* *}$ & 0.45 \\
\hline \multicolumn{10}{|l|}{ Validation } \\
\hline Social_cognition & 2.824 & 0.012 & $0.014^{*}$ & $0.008^{* *}$ & -1.13 & $0.010^{*}$ & -0.75 & 0.159 & - \\
\hline Attention & 0.249 & 0.965 & 0.965 & - & - & - & - & - & - \\
\hline Verbal_memory & 7.112 & $<0.001$ & $<0.001^{* * *}$ & $<0.001^{* * *}$ & -1.48 & $<0.001^{* * *}$ & -2.51 & 0.050 & - \\
\hline Visual_memory & 8.628 & $<0.001$ & $<0.001^{* * *}$ & $<0.001^{* * *}$ & 2.29 & $<0.001^{* * *}$ & 3.04 & 0.052 & - \\
\hline Salience & 3.533 & 0.001 & $0.001^{* *}$ & $0.008^{* *}$ & -1.12 & $<0.001^{* * *}$ & -1.03 & 0.578 & - \\
\hline
\end{tabular}

Demographic characteristics

Cognitively impaired patients showed significantly reduced number of years of education $\left(p_{\mathrm{fdr}}<0.001\right)$ and a significantly decreased female-to-male ratio $\left(p_{\mathrm{fdr}}=0.009\right)$ compared to $\mathrm{HC}$. Patients in the spared cluster showed significantly lower number of years of education $\left(p_{\mathrm{fdr}}=0.002\right)$ and lower female-tomale ratio $\left(p_{\mathrm{fdr}}<0.001\right)$ as compared to $\mathrm{HC}$. The number of patients recruited across sites differed significantly for the two clusters $\left(p_{\mathrm{fdr}}=0.046\right)$ and when comparing the impaired group and HC $\left(p_{\mathrm{fdr}}=0.014\right)$. Clusters did not differ regarding chlorpromazine equivalent level $\left(p_{\mathrm{fdr}}<0.100\right)$ and illness duration $\left(p_{\mathrm{fdr}}<0.440\right)$ (Table 3).

\section{Clinical characteristics}

Cognitively impaired patients showed significantly lower premorbid intelligence $\left(p_{\mathrm{fdr}}<0.001, d>1.04\right)$, lower GAF score in the last month $\left(p_{\mathrm{fdr}}=0.027, d=0.49\right)$, in the last year $\left(p_{\mathrm{fd}}=0.035, d=\right.$ $0.46)$ and lifetime $\left(p_{\mathrm{fdr}}=0.011, d=0.59\right)$ and lower GF scores at examination $\left(p_{\mathrm{fdr}}<0.045, d>0.43\right)$, last year $\left(p_{\mathrm{fdr}}<0.50, d>0.42\right)$ and across lifetime $\left(p_{\mathrm{fdr}}<0.024, d>0.51\right)$ when compared to patients in the spared cluster. Cognitively impaired patients showed significantly higher scores on the PANSS negative scale $\left(p_{\mathrm{fdr}}=0.003, d=-0.72\right)$ (Table S4 and Fig. 1B-E).

Validation sample

Observations in the validation sample were assigned to the impaired (impaired val,$N=13$ ) and spared (spared vali $N=40$ ) cluster of the discovery sample.

\section{Neurocognitive characteristics}

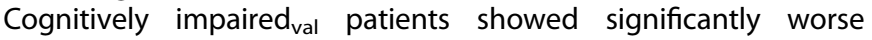
performance in social cognition $\left(p_{\mathrm{fdr}}=0.008, d=-1.13\right)$, processing speed $\left(p_{\mathrm{fdr}}<0.001, d=1.91\right)$, executive functioning $\left(p_{\mathrm{fdr}}=\right.$ $0.020, d=0.98)$, salience $\left(p_{\mathrm{fdr}}=0.008, d=-1.12\right)$ and verbal $\left(p_{\mathrm{fdr}}\right.$ $<0.001, d=-1.48)$ and visual memory $\left(p_{\mathrm{fdr}}<0.001, d=-2.29\right)$

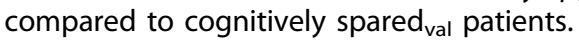

Cognitively impaired $d_{\text {val }}$ patients performed significantly worse regarding social cognition ( $p_{\mathrm{fdr}}=0.010, d=-0.75$ ), processing speed $\left(p_{\mathrm{fdr}}<0.001, d=2.48\right)$, executive functioning $\left(p_{\mathrm{fdr}}=0.023\right.$, $d=0.67)$, salience $\left(p_{\mathrm{fdr}}<0.001, d=-1.03\right)$ and verbal $\left(p_{\mathrm{fdr}}<0.001\right.$, $d=-2.51)$ and visual memory $\left(p_{\mathrm{fdr}}<0.001, d=3.04\right)$ when compared to $\mathrm{HC}$.

Cognitively spared $_{\text {val }}$ patients showed significantly reduced performance in processing speed $\left(p_{\mathrm{fdr}}=0.007, d=0.50\right)$ in comparison to $\mathrm{HC}$.

Demographic characteristics

Cognitively impaired ${ }_{\text {val }}$ patients showed no significant differences to cognitively spared $_{\text {val }}$ patients and HC. Cognitively spared $_{\text {val }}$ patients showed a significantly lower number of years of education $\left(p_{\mathrm{fdr}}=0.001\right)$ and lower female-to-male ratio $\left(p_{\mathrm{fdr}}=\right.$ 0.017) compared to HC. Clusters did not differ regarding chlorpromazine equivalent level $\left(p_{\mathrm{fdr}}=0.535\right)$ and illness duration $\left(p_{\mathrm{fdr}}=0.535\right)$ (Table 3$)$.

Clinical characteristics

Cognitively impaired $_{\text {val }}$ patients showed significantly lower premorbid intelligence $\left(p_{\mathrm{fdr}}<0.001, d=1.66\right)$ and lower GF scores for role functioning last year $\left(p_{\mathrm{fdr}}=0.042, d=0.87\right)$ and across life span $\left(p_{\mathrm{fdr}}=0.042, d=0.87\right)$ when compared to cognitively spared $_{\text {val }}$ patients (Table S4 and Fig. S5B-E).

\section{sMRI classification results}

A neuroanatomical SVM classification model discriminated the cognitively impaired patient group from $\mathrm{HC} \quad(B A C=60.1 \%$, sensitivity $=56.1 \%$, specificity $=64.1 \%, \quad N N D=5.0 ; p=0.01$ ) in the discovery sample. The classification model of the cognitively spared group against the $\mathrm{HC}(\mathrm{BAC}=55.4 \%$, sensitivity $=47.8 \%$, specificity $=63.1 \% ; \quad p=0.09$ ) and the cognitively spared group against the cognitively impaired group (BAC $=47.2 \%$, sensitivity $=31.7 \%$, specificity $=62.7 \% ; p=0.79$ ) remained nonsignificant (Fig. 2). 

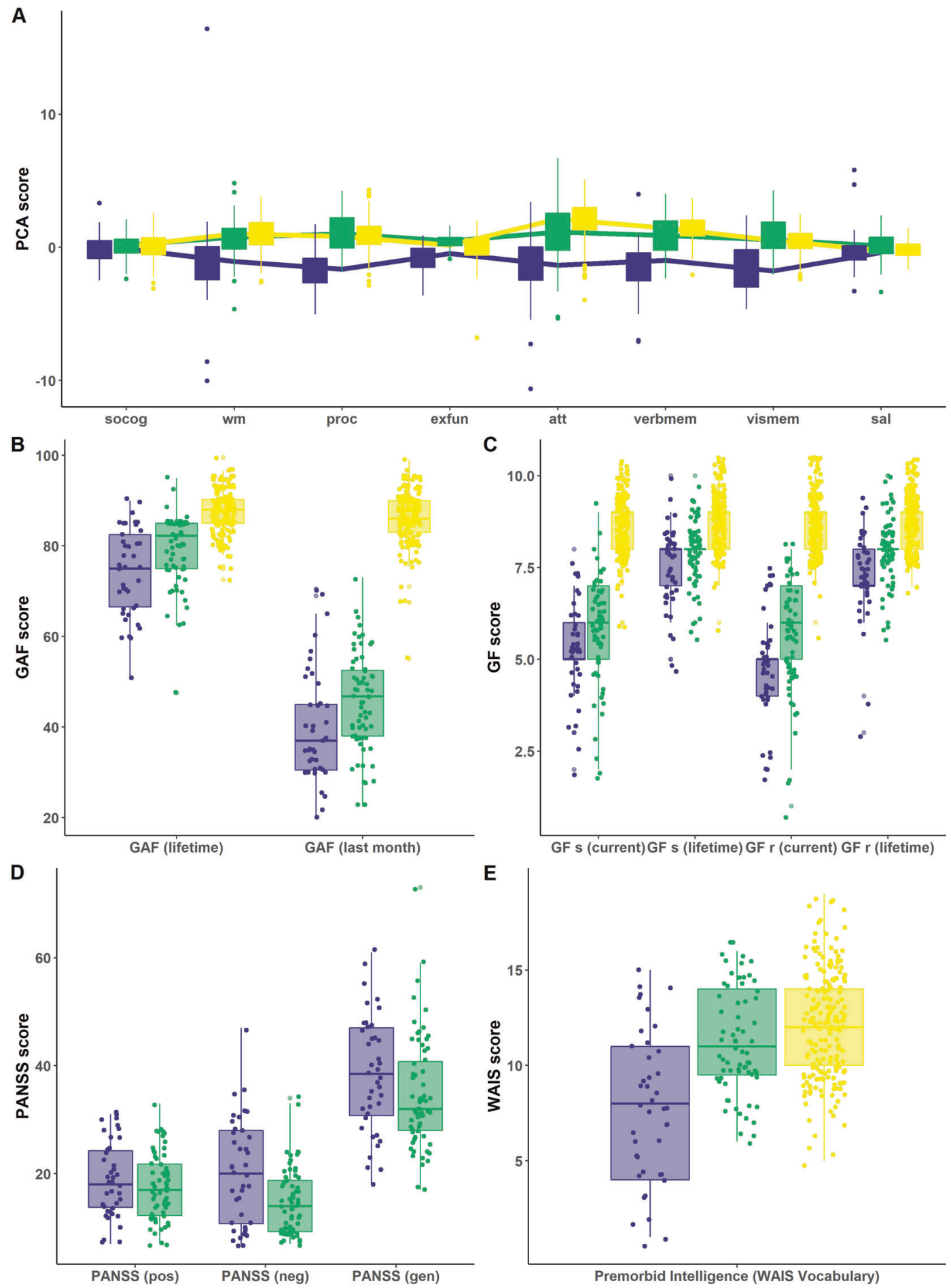

Fig. 1 Neuropsychological and clinical differences between clusters and HC in the discovery sample. Differences between the impaired (blue; $N=41$ ) and spared cluster (green; $N=67$ ) and HC (yellow; $N=195$ ) regarding A the neuropsychological PCA components, B the General Assessment of Functioning score (GAF), C the General Functioning score (GF), D the Positive and Negative Syndrom Scale (PANSS) and E Premorbid Verbal Intelligence are shown. A High PCA scores represent high performance. PCA scales for cognitive domains where high PCA scores represent low performance, are inverted. socog social cognition, wm working memory, proc processing speed, exfun executive functioning, att attention, verbmem verbal memory, vismem visual memory, sal salience.

The neuroanatomical signature between cognitively impaired ROP and HC group comprised both cortical and subcortical regions. Bilateral GMV increases associated with 'cognitively impaired ROP' status were predominantly found in basal ganglia and cerebellum and to a lesser extent in the middle frontal and inferior temporal gyrus. The unilateral GMV decreases were localized in the right superior frontal, supplementary motor areas and anterior cingulum. Left lateralized reductions were found in inferior occipital and orbito-frontal gyrus and superior temporal pole.

Increases in GMV associated with $\mathrm{HC}$ status were found bilaterally in the Heschl's gyrus, supramarginal gyrus, superior 
Table 3. Demographical effects between impaired and spared cluster and healthy controls in discovery and validation sample.

\begin{tabular}{|c|c|c|c|c|c|c|c|c|}
\hline & $\begin{array}{l}\text { Impaired } \\
\text { Mean (sd) }\end{array}$ & $\begin{array}{l}\text { Spared } \\
\text { Mean (sd) }\end{array}$ & \multicolumn{3}{|l|}{ Overall } & $\begin{array}{l}\text { Impaired vs. spared } \\
p \text { (FDR) }\end{array}$ & $\begin{array}{l}\text { Impaired vs. HC } \\
p \text { (FDR) }\end{array}$ & $\begin{array}{l}\text { Spared vs. HC } \\
p \text { (FDR) }\end{array}$ \\
\hline \multicolumn{9}{|l|}{ Discovery } \\
\hline Age & $23.5(4.3)$ & $25.8(5.4)$ & 2.015 & 0.106 & 0.109 & - & - & - \\
\hline Years of Education & $13.5(3.2)$ & $14.5(3.3)$ & 4.612 & $<0.001$ & $<0.001^{* * *}$ & 0.135 & $<0.001 * * *$ & $0.002^{* *}$ \\
\hline $\operatorname{Sex}^{\mathrm{a}}$ & female $=16$ & female $=19$ & 25.611 & $<0.001$ & $<0.001^{* * *}$ & 0.302 & $0.009^{* *}$ & $<0.001^{* * *}$ \\
\hline Ìllness duration in days ${ }^{c}$ & $163.66(153.82)$ & $192.43(205.69)$ & -0.770 & 0.440 & 0.440 & - & - & - \\
\hline Chlorpromazine equivalent $^{d}$ & $685.65(1596.42)$ & $196.95(125.38)$ & 1.940 & 0.052 & 0.100 & - & - & - \\
\hline \multicolumn{9}{|l|}{ Validation } \\
\hline $\mathrm{N}$ & 13 & 40 & & & & & & \\
\hline Age & $24.2(5.3)$ & $26.2(6.7)$ & 0.899 & 0.630 & 0.673 & - & - & - \\
\hline Years of Education & $14.3(3.7)$ & $14.0(3.5)$ & 3.594 & $<0.001$ & $0.001^{* *}$ & 0.858 & 0.081 & $0.001^{* *}$ \\
\hline $\begin{array}{l}H C \text { healthy control, } s d \text { standard } \\
\text { a'Nominal permutation test are u } \\
{ }^{\mathrm{b}} \text { Sites: Munich/Basel/Köln/Udine/ } \\
{ }^{\mathrm{C} D i f f e r e n c e ~ i n ~ t i m e ~ b e t w e e n ~ f i r s t ~} \\
{ }^{d} \text { Cumulative sum of chlorproma } \\
{ }^{*} p<0.05,{ }^{* *} p<0.01,{ }^{* * *} p<0.001 .\end{array}$ & $\begin{array}{l}\text { used; Fisher's exact } \\
\text { /Milan. } \\
\text { t fulfillment of psy } \\
\text { azine equivalents d }\end{array}$ & $\begin{array}{l}\text { t } p \text { value is repor } \\
\text { chotic diagnosis } \\
\text { divided by the }\end{array}$ & $r$ of $d$ & streates & Clinic & $\log _{0} 0$ & and & ation. \\
\hline
\end{tabular}

temporal gyrus and rolandic operculum. Further, bilateral increases in GMV were located in superior frontal and middle occipital regions, precuneus, in the cingulum and parahippocampal gyrus. The unilateral GMV increases were shown in left inferior frontal areas and cerebellum alongside with GMV increases in right superior parietal regions and angular gyrus, inferior orbital gyrus and hippocampus.

\section{DISCUSSION}

Our study reveals two cognitively and clinically distinct neurocognitive subgroups in ROP patients in line with previously reported cognitive subgroups in chronic SZ patients [8-11]. To the best of our knowledge, this is the first study showing altered cognitive, clinical and neuroanatomical features, using unsupervised ML methods, in the early stages of psychosis when patients are minimally affected by antipsychotic medication. We obtain a largely impaired and a spared subgroup and validate both in an independent behavioral data set of ROP patients. Whilst the applied neuroanatomical classification analysis was successful in distinguishing the cognitively and clinically impaired cluster from $\mathrm{HC}$, it revealed no statistical differences between the spared subgroup and HC.

The current study found an impaired cluster presenting with more profound cognitive deficits in the domains of processing speed, working memory, executive functioning, attention and visual and verbal memory in comparison to HC. The spared cluster shows impairments in attention and verbal memory relative to $\mathrm{HC}$, however, a similar performance in working memory, processing speed and social cognition. Conversely, this cluster shows increased performance in executive functioning, salience and visual memory relative to $\mathrm{HC}$ (Fig. 1 and Table 2). Increased performance in a psychosis subgroup relative to $\mathrm{HC}$ has been reported in a previous study [47]. The presence of cognitively and functionally preserved individuals in one subgroup might have been easier to identify due to our minimally medicated recent onset sample in comparison to previously employed chronic patient cohorts [8-11].
Analysis of the cognitive clusters' clinical characteristics revealed premorbid general functioning $[8,10,11]$, social and occupational functioning $[9,11]$ difficulties in the impaired group which were less present in the spared group (Supplementary Table S4). In line with prior studies, we confirmed a higher level of negative symptoms in impaired ROP patients as compared to the spared ROP patients $[8,9]$ (Supplementary Table S4). Importantly, though making a major contribution to the cluster solution, cognitive subgroups were not entirely explained by premorbid intelligence (Supplementary Materials and Methods).

Similar as in the discovery sample, we found reduced performance in processing speed, executive functioning and verbal and visual memory alongside impaired premorbid intelligence level and partially impaired functioning for impaired val $_{\text {la }}$ patients when compared to spared $_{\text {val }}$ patients and $\mathrm{HC}$ of the independent behavioral data set. The concordance on verbal memory and processing speed deficits between impaired patients across both samples supports recent efforts of the second phase of the North American Psychosis Longitudinal Study-II that generated a risk calculator for transition to psychosis integrating both domains in its prediction model [48].

Our classification analysis reliably showed patterns of GMV increases associated with impaired-cluster status predominantly in the subcortical area of putamen [13] while we observed smaller increases in cortical areas [49]. Basal ganglia enlargement seems to occur in medication-naive populations with clinical and genetic risk [50]. As our ROP patients were newly exposed to antipsychotic treatment, larger basal ganglia appear to reflect striatal hyperdopaminergia possibly related to acute psychotic symptoms [51]. In previous studies, unaffected family members have also shown larger putamen [51]. However, HC have shown increases in frontotemporo-parietal cortical regions with an emphasis on Heschl's gyrus [52] and parahypocampal areas [53] which are particularly prone to GMV loss in psychosis $[16,49]$.

Previous studies propose a preadolescent decline trajectory for SZ, characterized by impaired premorbid intelligence, reduced general cognition at illness onset and lower level of occupational functioning [11]. First, impaired patients show high levels of 

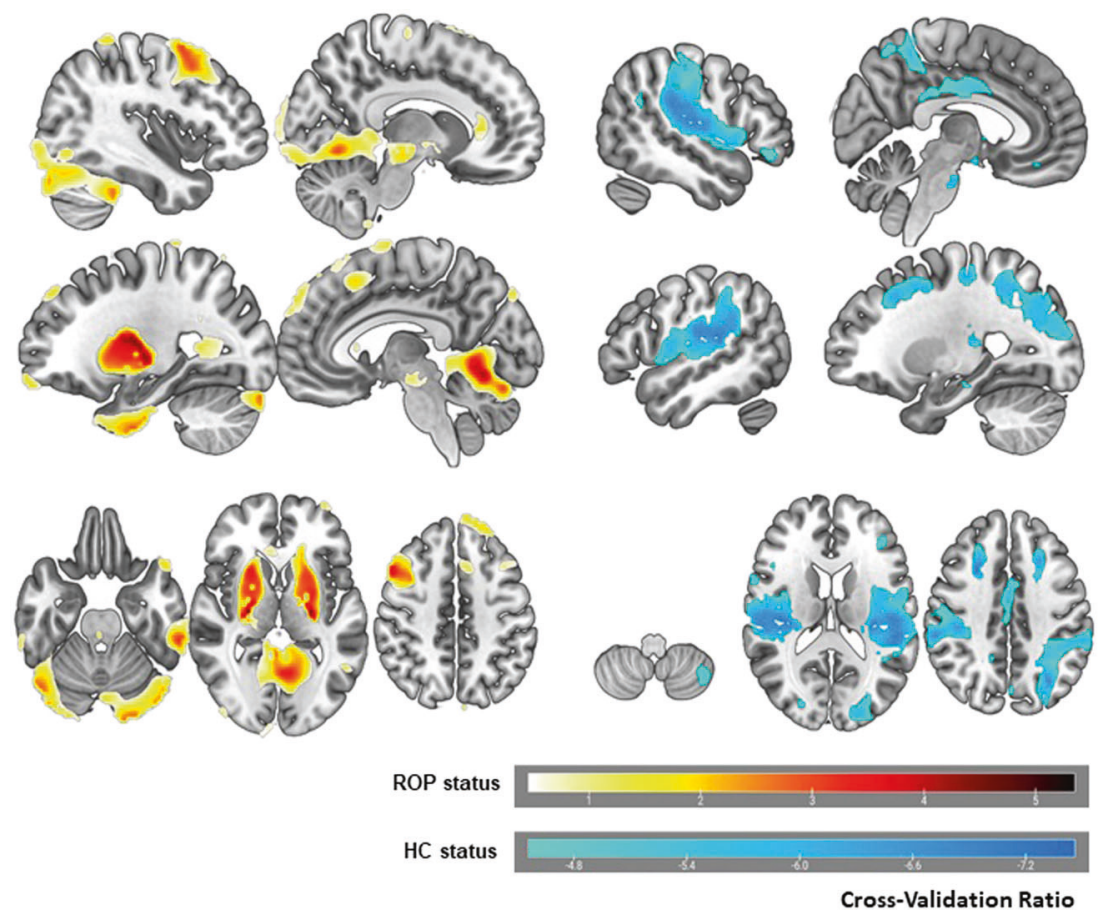

Fig. 2 Reliability of predictive voxels for the impaired vs. HC classification model. Voxel-wise reliabilities are represented by the crossvalidation ratio. Warm colors represent the $10 \%$ most reliable voxels predicting impaired ROP status, i.e., areas with increased gray matter (GM) in ROP. Cool colors represent the $10 \%$ most reliable voxels predicting HC status, i.e., areas with increased GM in HC. Left and right hemisphere are reversed.

negative symptoms $[8,9]$ and gradual differences in social and occupational functioning in comparison to spared subgroup and HC. Second, studies demonstrate developmental lags relative to same-aged HC [54] in CHR individuals who go on to develop fullblown psychosis. Large cohort studies in CHR [55] implicate that immediate verbal learning, memory and processing speed are the most relevant domains for prediction of transition to psychosis. Those domains are significantly reduced in our impaired subgroup (Supplementary Fig. S9) and replicate in the validation sample. Third, previous cross-sectional findings on ultra-high risk (UHR) individuals who later transitioned to psychosis reported reduced GMV in prefrontal areas, temporal gyrus and cerebellum relative to $\mathrm{HC}$ and to UHR who did not transition to psychosis, respectively $[56,57]$. In the current study, the impaired subgroup shows a significant neuroanatomical signature relative to $\mathrm{HC}$. The presence of GMV reduction, despite the absence of chronicity and longterm medication effects, suggests these brain alterations may have emerged before the onset of florid psychotic symptoms. Finally, both behavioral and imaging effects persist after controlling for differences across subgroups regarding age, sex, educational years, study site and group sizes. In addition, post hoc examination of the relationship between decision scores of the 'impaired subgroup vs $\mathrm{HC}^{\prime}$ neuroimaging classification model and study site ensures that our classification model is not mainly driven by site-specific scanner differences (Supplementary Materials and Methods).

The current study has several limitations. First, the applied neuropsychological tasks differed from the MCCB [25] and cognitive domains, e.g., social cognition and executive functioning, were underrepresented in comparison to other tests (Table S1). Second, we could only partially replicate the effects of the discovery cluster solution. This might be due to differences in sample characteristics and sizes (Table 1) or the monocentric characteristic of the validation sample. Third, while we suggest that the characteristics of the impaired subgroup align with early maladaptive processes as proposed in the neurodevelopmental hypothesis [1], our assessment of functioning is retrospective and cross-sectional. Future studies would benefit from a longitudinal design providing a more comprehensive answer. Fourth, as crosssite data acquisition differences arise as key issues in multi-center studies [58], we accounted for such effects in both behavioral and neuroimaging analysis. However, an effect of an unbalanced distribution of participants between subgroups and $\mathrm{HC}$ on our cluster findings cannot be ruled out entirely.

Cognitive and clinical differences in the psychosis subgroups of the discovery sample support the idea of distinct trajectories in early stages of the disease [5]. In accordance with this finding is the neurobiological separability of cognitively impaired patients from HC. Early detection of psychosis subgroups could help to tailor early interventions for ROP patients with likely stronger neurodevelopmental character of psychosis. A prime candidate to achieve this might be neurocognitive intervention showing positive effect on cognition and functioning in patients suffering from SZ [7]. Further studies should investigate if the suggested clusters are shared between different phenotypes, particularly affective psychosis, and if common transdiagnostic pathways can be found for patients with cognitive impairments.

\section{FUNDING AND DISCLOSURE}

This work was supported in analysis and writing of the manuscript by the European Union-FP7 project PRONIA ("Personalized Prognostic Tools for Early Psychosis Management", grant number 602152). JW was partly supported by the NARSAD Young Investigator Award of LK through the Brain and Behavior Research Foundation (grant number 28474). NK, JK and RKRA are currently honorary speakers for Otsuka/Lundbeck. RU achieved grants from Medical Research Council, grants from the National Institute for Health Research, and personal fees from Sunovion. The remaining authors including members of the PRONIA consortium have nothing to disclose. All procedures contributing to this work comply with the ethical standards of the relevant national and 
institutional committees on human experimentation and with the Helsinki Declaration of 1975, as revised in 2008.

\section{DATA AVAILABILITY}

The data models that support the findings of this study are available on request from the corresponding author [LK-I and NK]. The data are not publicly available due to ethical restrictions.

\section{AUTHOR CONTRIBUTIONS}

Analysis or interpretation of data: JW, LK-I, NK, DBD, JK. Concept and design: LK-I, NK, DBD, JK, RU, RKRS, EM, SJW, PB, Borgwardt. Drafting of the manuscript: JW, LK-I. Critical revision of the manuscript for important intellectual content: all authors. Data acquisition, analysis, quality control and MRI support: $\mathrm{SSH}, \mathrm{AR}$, OFO, LA, SVS, CB, MG, $A F, M P, J B$ and the PRONIA consortium.

\section{FUNDING}

Open Access funding enabled and organized by Projekt DEAL.

\section{DATA AVAILABILITY}

The data models that support the findings of this study are available on request from the corresponding author [LK-I and NK]. The data are not publicly available due to ethical restrictions.

\section{ADDITIONAL INFORMATION}

Supplementary information The online version contains supplementary material available at https://doi.org/10.1038/s41386-021-00963-1.

Publisher's note Springer Nature remains neutral with regard to jurisdictional claims in published maps and institutional affiliations.

\section{REFERENCES}

1. Howes OD, Murray RM. Schizophrenia: an integrated sociodevelopmentalcognitive model. Lancet. 2014;383:1677-87.

2. Reichenberg AA. The assessment of neuropsychological functioning in schizophrenia. Dialogues Clin Neurosci. 2010;12:383.

3. Sheffield JM, Karcher NR, Barch DM. Cognitive deficits in psychotic disorders: a lifespan perspective. Neuropsychol Rev. 2018;28:509-33.

4. Vita A, De Peri L, Silenzi C, Dieci M. Brain morphology in first-episode schizophrenia: a meta-analysis of quantitative magnetic resonance imaging studies. Schizophr Res. 2006;82:75-88.

5. Lewandowski KE. Genetically, developmentally, and clinically distinct cognitive subtypes in schizophrenia: A tale of three trajectories. Am J Psychiatry. 2020;177:282-4.

6. Kahn RS, Keefe RS. Schizophrenia is a cognitive illness: time for a change in focus. JAMA Psychiatry. 2013;70:1107-12.

7. Kambeitz-llankovic L, Betz LT, Dominke C, Haas SS, Subramaniam K, Fisher M, et al. Multi-outcome meta-analysis (MOMA) of cognitive remediation in schizophrenia: Revisiting the relevance of human coaching and elucidating interplay between multiple outcomes. Neurosci Biobehav Rev. 2019;107:828-45.

8. Green M, Cairns M, Wu J, Dragovic M, Jablensky A, Tooney P, et al. Genome-wide supported variant MIR137 and severe negative symptoms predict membership of an impaired cognitive subtype of schizophrenia. Mol Psychiatry. 2013;18:774-80.

9. Lewandowski K, Sperry S, Cohen B, Öngür D. Cognitive variability in psychotic disorders: a cross-diagnostic cluster analysis. Psychol Med. 2014:44:3239-48.

10. Van Rheenen TE, Cropley V, Zalesky A, Bousman C, Wells R, Bruggemann J, et al. Widespread volumetric reductions in schizophrenia and schizoaffective patients displaying compromised cognitive abilities. Schizophr Bull. 2018:44:560-74.

11. Dickinson D, Zaidman SR, Giangrande EJ, Eisenberg DP, Gregory MD, Berman KF. Distinct Polygenic Score Profiles in Schizophrenia Subgroups with Different Trajectories of Cognitive Development. Am J Psychiatry. 2020;177:298-307.

12. Gould IC, Shepherd AM, Laurens KR, Cairns MJ, Carr VJ, Green MJ. Multivariate neuroanatomical classification of cognitive subtypes in schizophrenia: a support vector machine learning approach. Neuroimage Clin. 2014;6:229-36.

13. Chand GB, Dwyer DB, Erus G, Sotiras A, Varol E, Srinivasan D, et al. Two distinct neuroanatomical subtypes of schizophrenia revealed using machine learning. Brain. 2020:143:1027-38.
14. Dwyer DB, Cabral C, Kambeitz-llankovic L, Sanfelici R, Kambeitz J, Calhoun V, Falkai $\mathrm{P}$, Pantelis $\mathrm{C}$, Meisenzahl E, Koutsouleris N. Brain subtyping enhances the neuroanatomical discrimination of schizophrenia. Schizophrenia Bull. 2018;44: 1060-9.

15. Lewandowski K, Cohen B, Öngur D. Evolution of neuropsychological dysfunction during the course of schizophrenia and bipolar disorder. Psychol Med. 2011;41: 225-41.

16. Haijma SV, Van Haren N, Cahn W, Koolschijn PCM, Hulshoff Pol HE, Kahn RS. Brain volumes in schizophrenia: a meta-analysis in over 18000 subjects. Schizophr Bull. 2013;39:1129-38.

17. Dwyer DB, Falkai $P$, Koutsouleris N. Machine learning approaches for clinical psychology and psychiatry. Annu Rev Clin Psychol. 2018;14:91-118.

18. Arlington V, American Psychiatric Association. Diagnostic and statistical manual of mental disorders. Am Psychiatr Assoc. 2013;5:612-3.

19. Koutsouleris N, Kambeitz-llankovic L, Ruhrmann S, Rosen M, Ruef A, Dwyer DB, et al. Prediction models of functional outcomes for individuals in the clinical high-risk state for psychosis or with recent-onset depression: a multimodal, multisite machine learning analysis. JAMA Psychiatry. 2018;75:1156-72.

20. Miller TJ, McGlashan TH, Rosen JL, Cadenhead K, Ventura J, McFarlane W, et al. Prodromal assessment with the structured interview for prodromal syndromes and the scale of prodromal symptoms: predictive validity, interrater reliability, and training to reliability. Schizophr Bull. 2003;29:703-15.

21. Schultze-Lutter F, Addington J, Ruhrmann S, Klosterkötter J. Schizophrenia proneness instrument, adult version (SPI-A). Rome: Giovanni Fioriti; 2007.

22. Hall RC. Global assessment of functioning: a modified scale. Psychosomatics. 1995;36:267-75.

23. Cornblatt BA, Auther AM, Niendam T, Smith CW, Zinberg J, Bearden CE, et al. Preliminary findings for two new measures of social and role functioning in the prodromal phase of schizophrenia. Schizophr Bull. 2007;33:688-702.

24. Kay SR, Fiszbein A, Opler LA. The positive and negative syndrome scale (PANSS) for schizophrenia. Schizophr Bull. 1987;13:261-76.

25. Nuechterlein KH, Green MF, Kern RS, Baade LE, Barch DM, Cohen JD, et al. The MATRICS consensus cognitive battery, part 1: test selection, reliability, and validity. Am J Psychiatry. 2008;165:203-13.

26. Osterrieth P. The test of copying a complex figure: a contribution to the study of perception and memory. Arch Psychol. 1944;30:206-356.

27. Nowicki S, Duke MP. Manual for the receptive tests of the diagnostic analysis of nonverbal accuracy 2 (DANVA2). Atlanta, GA: Department of Psychology, Emory University; 2008.

28. Wechsler D. Manual for the Wechsler Adult Intelligence Scale. Manual for the Wechsler Adult Intelligence Scale. Oxford, England: Psychological Corp.; 1955. p. vi,110-vi,110.

29. Petrides $M$, Milner B. Deficits on subject-ordered tasks after frontal-and temporallobe lesions in man. Neuropsychologia. 1982;20:249-62.

30. Ruff RM, Light RH, Parker SB, Levin HS. Benton controlled oral word association test: reliability and updated norms. Arch Clin Neuropsychol. 1996;11: 329-38.

31. Army individual test battery. Manual of Directions and Scoring. Washington, DC: War Department, Adjunct General's Office; 1994.

32. Schmidt M. Rey auditory verbal learning test: a handbook. Los Angeles, CA: Western Psychological Services; 1996.

33. Eliason MJ, Richman LC. The continuous performance test in learning disabled and nondisabled children. J Learn Disabilities. 1987;20:614-9.

34. Kapur S. Psychosis as a state of aberrant salience: a framework linking biology, phenomenology, and pharmacology in schizophrenia. Am J Psychiatry. 2003;160:13-23.

35. Koutsouleris N, Meisenzahl EM, Borgwardt S, Riecher-Rössler A, Frodl T, Kambeitz $J$, et al. Individualized differential diagnosis of schizophrenia and mood disorders using neuroanatomical biomarkers. Brain 2015;138:2059-73.

36. Lloyd S. Least squares quantization in PCM. IEEE Trans Inf Theory. 1982;28: 129-37.

37. Hennig C. Cluster-wise assessment of cluster stability. Comput Stat Data Anal. 2007;52:258-71.

38. Benjamini $\mathrm{Y}$, Hochberg $\mathrm{Y}$. Controlling the false discovery rate: a practical and powerful approach to multiple testing. J R Stat Soc Ser B Stat Methodol. 1995:57:289-300.

39. Hennig C. Flexible procedures for clustering. R package version. 2020;2:2-5.

40. Leisch F. A toolbox for k-centroids cluster analysis. Comput Stat Data Anal. 2006;51:526-44.

41. Hothorn T, Hornik K, Van De Wiel MA, Zeileis A. A lego system for conditional inference. Am Stat. 2006;60:257-63.

42. Mangiafico S. rcompanion: functions to support extension education program evaluation. $\mathrm{R}$ package version. Vol. 1. 2017. https://CRAN.R-project.org/ package $=$ rcompanion 
43. Golland P, Fischl B. Permutation tests for classification: Towards statistical significance in image-based studies. Lect Notes Comput Sci (including Subser Lect Notes Artif Intell Lect Notes Bioinformatics). 2003;2732:330-41.

44. Caliński T, Harabasz J. A dendrite method for cluster analysis. Commun Stat Theory Methods. 1974;3:1-27.

45. Rousseeuw PJ. Silhouettes: a graphical aid to the interpretation and validation of cluster analysis. J Comput Appl Math. 1987;20:53-65.

46. Jaccard P. Nouvelles recherches sur la distribution florale. Bull Soc Vaud Sci Nat. 1908;44:223-70.

47. Karantonis JA, Rossell SL, Carruthers SP, Sumner P, Hughes M, Green MJ, Pantelis C, Burdick KE, Cropley V, Van, Rheenen TE. Cognitive validation of crossdiagnostic cognitive subgroups on the schizophrenia-bipolar spectrum. J Affect Disord. 2020;266(Apr):710-21.

48. Cannon TD, Yu C, Addington J, Bearden CE, Cadenhead KS, Cornblatt BA, et al. An individualized risk calculator for research in prodromal psychosis. Am J Psychiatry. 2016;173:980-8.

49. van Erp TG, Hibar DP, Rasmussen JM, Glahn DC, Pearlson GD, Andreassen OA et al. Subcortical brain volume abnormalities in 2028 individuals with schizophrenia and 2540 healthy controls via the ENIGMA consortium. Mol Psychiatry. 2016;21:547-53.

50. Gong Q, Scarpazza C, Dai J, He M, Xu X, Shi Y, et al. A transdiagnostic neuroanatomical signature of psychiatric illness. Neuropsychopharmacol. 2019;44: 869-75.

51. Howes OD, Kapur S. The dopamine hypothesis of schizophrenia: version III-the final common pathway. Schizophr Bull. 2009;35:549-62.

52. Honea R, Crow TJ, Passingham D, Mackay CE. Regional deficits in brain volume in schizophrenia: a meta-analysis of voxel-based morphometry studies. Am J Psychiatry. 2005;162:2233-45.

53. Lieberman J, Girgis R, Brucato G, Moore H, Provenzano F, Kegeles L, et al. Hippocampal dysfunction in the pathophysiology of schizophrenia: a selective review and hypothesis for early detection and intervention. Mol Psychiatry. 2018;23:1764-72.
54. Kambeitz-Ilankovic L, Haas SS, Meisenzahl E, Dwyer DB, Weiske J, Peters H, et al. Neurocognitive and neuroanatomical maturation in the clinical high-risk states for psychosis: a pattern recognition study. Neuroimage Clin. 2019;21:101624.

55. Allott K, Wood SJ, Yuen HP, Yung AR, Nelson B, Brewer WJ, et al. Longitudinal cognitive performance in individuals at ultrahigh risk for psychosis: a 10-year follow-up. Schizophr Bull. 2019;45:1101-11.

56. Koutsouleris N, Meisenzahl EM, Davatzikos C, Bottlender R, Frodl T, Scheuerecker $\mathrm{J}$, et al. Use of neuroanatomical pattern classification to identify subjects in at-risk mental states of psychosis and predict disease transition. Arch Gen Psychiatry. 2009;66:700-12.

57. Cannon TD. Brain biomarkers of vulnerability and progression to psychosis. Schizophr Bull 2016;42:S127-32.

58. Chen J, Liu J, Calhoun VD, Arias-Vasquez A, Zwiers MP, Gupta CN, Franke B, Turner JA. Exploration of scanning effects in multi-site structural MRI studies. J Neurosci Methods. 2014;230:37-50.

\begin{abstract}
Open Access This article is licensed under a Creative Commons Attribution 4.0 International License, which permits use, sharing, adaptation, distribution and reproduction in any medium or format, as long as you give appropriate credit to the original author(s) and the source, provide a link to the Creative Commons license, and indicate if changes were made. The images or other third party material in this article are included in the article's Creative Commons license, unless indicated otherwise in a credit line to the material. If material is not included in the article's Creative Commons license and your intended use is not permitted by statutory regulation or exceeds the permitted use, you will need to obtain permission directly from the copyright holder. To view a copy of this license, visit http://creativecommons. org/licenses/by/4.0/.
\end{abstract}

(c) The Author(s) 2021

\section{THE PRONIA CONSORTIUM}

Nikolaos Koutsouleris ${ }^{3,18,19}$, Lana Kambeitz-llankovic ${ }^{1,3 *}$, Mark Sen Dong ${ }^{3}$, Anne Erkens ${ }^{3}$, Eva Gussmann ${ }^{3}$, Shalaila Haas ${ }^{2}$, Alkomiet Hasan $^{20}$, Claudius Hoff ${ }^{3}$, Ifrah Khanyaree ${ }^{3}$, Aylin Melo ${ }^{3}$, Susanna Muckenhuber-Sternbauer ${ }^{3}$, Janis Kohler ${ }^{3}$, Oemer Faruk Oeztuerk ${ }^{3,4}$, David Popovic ${ }^{3}$, Nora Penzel ${ }^{1}$, Adrian Rangnick ${ }^{3}$, Sebastian von Saldern ${ }^{3}$, Rachele Sanfelici ${ }^{3,21}$, Moritz Spangemacher ${ }^{3}$, Ana Tupac ${ }^{3}$, Maria Fernanda Urquijo ${ }^{3}$, Johanna Weiske, Julian Wenzel ${ }^{1}$, Antonia Wosgien ${ }^{3}$, Joseph Kambeitz (D) ${ }^{1}$, Stephan Ruhrmann ${ }^{1}$, Marlene Rosen ${ }^{1}$, Linda Betz ${ }^{1}$, Theresa Haidl ${ }^{1}$, Karsten Blume ${ }^{1}$, Mauro Seves ${ }^{1}$, Nathalie Kaiser ${ }^{1}$, Tanja Pilgram ${ }^{1}$, Thorsten Lichtenstein ${ }^{1}$, Christiane Woopen ${ }^{1}$, Stefan Borgwardt ${ }^{11}$, Christina Andreou ${ }^{22}$, Laura Egloff ${ }^{22}$, Fabienne Harrisberger ${ }^{22}$, Claudia Lenz ${ }^{22}$, Letizia Leanza ${ }^{22}$, Amatya Mackintosh ${ }^{22}$, Renata Smieskova ${ }^{22}$, Erich Studerus ${ }^{22}$, Anna Walter ${ }^{22}$, Sonja Widmayer ${ }^{22}$, Rachel Upthegrove ${ }^{14,15}$, Stephen J. Wood ${ }^{14,16,17}$, Katharine Chisholm ${ }^{15}$, Chris Day ${ }^{15}$, Sian Lowri Griffiths ${ }^{15}$, Mariam Iqbal ${ }^{15}$, Paris Lalousis ${ }^{15}$, Mirabel Pelton ${ }^{15}$, Pavan Mallikarjun ${ }^{15}$, Alexandra Stainton ${ }^{15}$, Ashleigh Lin ${ }^{15}$, Raimo K. R. Salokangas $\mathbb{D}^{13}$, Alexander Denissoff ${ }^{13}$, Anu Ellila ${ }^{13}$, R. N. Tiina From ${ }^{13}$, Markus Heinimaa $^{13}$, Tuula Ilonen ${ }^{13}$, Paivi Jalo ${ }^{13}$, R. N. Heikki Laurikainen ${ }^{13}$, Maarit Lehtinen ${ }^{13}$, R. N. Antti Luutonen ${ }^{13}$, Akseli Makela ${ }^{13}$, Janina Paju $^{13}$, Henri Pesonen ${ }^{13}$, Reetta-Liina Armio (Saila) ${ }^{13}$, Elina Sormunen ${ }^{13}$, Anna Toivonen ${ }^{13}$, Otto Turtonen ${ }^{13}$, Ana Beatriz Solana ${ }^{23}$, Manuela Abraham ${ }^{23}$, Nicolas Hehn ${ }^{23}$, Timo Schirmer ${ }^{23}$, Paolo Brambilla (iD ${ }^{7,8}$, Carlo Altamura ${ }^{7,8}$, Marika Belleri ${ }^{7,8}$, Francesca Bottinelli ${ }^{7,8}$, Adele Ferro ${ }^{7,8}$, Marta Re ${ }^{7,8}$, Emiliano Monzani ${ }^{24}$, Mauro Percudani ${ }^{24}$, Maurizio Sberna ${ }^{24}$, Armando D'Agostino ${ }^{25}$, Lorenzo Del Fabro ${ }^{25}$, Villa San Benedetto Menni ${ }^{25}$, Giampaolo Perna ${ }^{25}$, Maria Nobile ${ }^{25}$, Alessandra Alciati ${ }^{25}$, Matteo Balestrieri ${ }^{26}$, Carolina Bonivento ${ }^{6}$, Giuseppe Cabras ${ }^{26}$, Franco Fabbro ${ }^{26}$, Marco Garzitto ${ }^{6}$, Sara Piccin ${ }^{6}$, Alessandro Bertolino ${ }^{5}$, Giuseppe Blasi ${ }^{5}$, Linda A. Antonucci ${ }^{3,5}$, Giulio Pergola $^{5}$, Grazia Caforio ${ }^{5}$, Leonardo Faio ${ }^{5}$, Tiziana Quarto ${ }^{5}$, Barbara Gelao ${ }^{5}$, Raffaella Romano ${ }^{5}$, lleana Andriola ${ }^{5}$, Andrea Falsetti ${ }^{5}$, Marina Barone $^{5}$, Roberta Passatiore ${ }^{5}$, Marina Sangiuliano ${ }^{5}$, Rebekka Lencer ${ }^{27}$, Marian Surman ${ }^{27}$, Olga Bienek ${ }^{27}$, Georg Romer ${ }^{27}$, Udo Dannlowski $^{27}$, Eva Meisenzahl ${ }^{12}$, Frauke Schultze-Lutter ${ }^{12}$, Christian Schmidt-Kraepelin ${ }^{12}$, Susanne Neufang ${ }^{12}$, Alexandra Korda ${ }^{12}$ and Henrik Rohner ${ }^{12}$

\footnotetext{
${ }^{20}$ Department for Psychiatry, Psychotherapy und Psychosomatics, University of Augsburg, Augsburg, Germany. ${ }^{21}$ Max Planck School of Cognition, Stephanstrasse 1a, Leipzig,

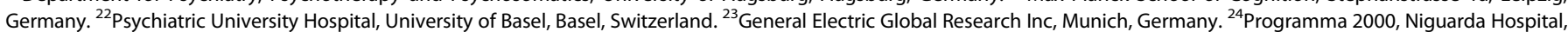
Milan, Italy. ${ }^{25}$ San Paolo Hospital, Milan, Italy. ${ }^{26}$ Department of Medical Area, University of Udine, Udine, Italy. ${ }^{27}$ Department of Psychiatry and Psychotherapy, Westfaelische Wilhelms-University Muenster, Muenster, Germany.
} 